\title{
Clinical, radiological, and biochemical characteristics in patients with diseases mimicking polymyalgia rheumatica
}

This article was published in the following Dove Press journal:

Clinical Interventions in Aging

30 September 2009

Number of times this article has been viewed

\author{
Hidekatsu Yanai ${ }^{1,2}$ \\ Hiroshi Yoshida ${ }^{2,3}$ \\ Norio Tada ${ }^{1,2}$ \\ 'Division of General Medicine, \\ Department of Internal Medicine, \\ The Jikei University School of \\ Medicine, Kashiwa, Japan; ${ }^{2}$ Institute \\ of Clinical Medicine and Research, \\ the Jikei University School of \\ Medicine, Chiba, Japan; ${ }^{3}$ Department \\ of Laboratory Medicine, The Jikei \\ University School of Medicine, \\ Kashiwa, Japan
}

\begin{abstract}
To find out clues to differentiate between polymyalgia rheumatica (PMR) and other diseases that mimic PMR. We studied Japanese patients with PMR $(n=7)$, pseudogout $(n=1)$, remitting seronegative symmetrical synovitis with pitting edema (RS3PE) syndrome ( $n=1)$, and post-infectious polyarthritis $(\mathrm{n}=1)$. The distribution of inflammation in patients was evaluated using a gallium-67 scintigraphy. We measured serum C-reactive protein (CRP), matrix metalloproteinase-3 (MMP-3), and vascular endothelial growth factor (VEGF) in patients before and after treatment. Further, we compared the clinical course of PMR with that of other diseases that mimic PMR. Patients with pseudogout, RS3PE syndrome, post-infectious polyarthritis manifested similar changes in scintigraphic findings and serum CRP, MMP-3, and VEGF levels to PMR before the treatment. A significant reduction in serum CRP levels at one week after use of nonsteroidal anti-inflammatory drugs (NSAIDs) is a good clue to differentiate pseudogout and post-infectious polyarthritis from PMR. Chondrocalcinosis in the radiographs of joints is also effective to differentiate pseudogout from PMR. A small reduction of CRP levels after NSAIDs use and promptly ameliorated CRP and symptoms by a low-dose steroid therapy, which was commonly observed in patients with PMR, were also found in a patient with RS3PE syndrome. Pitting edema of the back of hands and gallium uptake in metacarpophalangeal (MCP) joints were useful to differentiate RS3PE syndrome from PMR. In conclusion, pseudogout, RS3PE syndrome, post-infectious polyarthritis should be included in the spectrum of diseases mimicking PMR. A promptly decreased serum CRP level by NSAIDs is a good clue to differentiate pseudogout and post-infectious polyarthritis from PMR. Pitting edema of the back of hands and symmetric gallium uptake in MCP joints are characteristic for RS3PE syndrome.
\end{abstract}

Keywords: gallium-67 scintigraphy, polymyalgia rheumatica, pseudogout, post-infectious polyarthritis, RS3PE syndrome

\section{Introduction}

Polymyalgia rheumatica (PMR) is an inflammatory rheumatic disease in elderly people. It is a common indication for a long term treatment with steroid in patients based in the community. Although the cause of PMR is largely unknown, the presence of articular synovitis in PMR has been proven by synovial biopsy. ${ }^{1}$ Recently, we have reported that symmetrical gallium-67 uptake in the shoulders and elevated serum matrix metalloproteinase-3 (MMP-3) and vascular endothelial growth factor (VEGF) levels as the markers for synovitis in patients with PMR. ${ }^{2}$ Although bilateral aching and stiffness of shoulders and pelvic girdle of acute or subacute onset are very characteristic for PMR, several other diseases can be present with similar symptoms. Here, we studied a gallium scintigraphic findings, and serum C-reactive protein (CRP), MMP-3 and
Correspondence: Hidekatsu Yanai Division of General Medicine, Department of Internal Medicine, The Jikei University School of Medicine, Kashiwa, Japan

Tel +8I 47164 IIII

Fax $+8|47| 64$ II26

Email yanaih@jikei.ac.jp 
VEGF levels in patients with diseases that mimic PMR. Further, we discuss clues to differentiate between PMR and other diseases that mimic PMR.

\section{Methods}

We studied Japanese patients with PMR $(n=7)$, pseudogout $(\mathrm{n}=1)$, remitting seronegative symmetrical synovitis with pitting edema (RS3PE) syndrome $(n=1)$, and post-infectious polyarthritis $(n=1)$. Diagnosis for PMR had been done using the Hunder's criteria. ${ }^{3}$ The distribution of inflammation in patients was evaluated using a gallium-67 scintigraphy whole body scan before the treatment. We measured serum CRP, MMP-3, and VEGF levels as the markers for a synovial inflammation before and after the treatment. ${ }^{4,5}$ Serum CRP and MMP-3 concentrations were measured by polyclonal antibodybased (Dade Behring, Marburg, Germany) and monoclonal antibody-based (Daiichi Fine Chemical, Co., Ltd., Toyama, Japan) latex agglutination method, respectively. Serum concentrations of $\mathrm{VEGF}_{165}$ (major form of VEGF) were analyzed by enzyme linked immunosorbent assay (ELISA; VEGF ${ }_{165}$, R\&D Systems, Inc, Minneapolis, MN, USA).

\section{Results}

\section{Patients with PMR}

Case P is a 64-year-old woman with PMR (Figure 1P). Symmetrical and bilateral gallium uptake in shoulders, pelvic girdle, and knees were observed. Age of all patients with PMR were over 50 years (Table 1). Serum CRP (normal range, $<0.3 \mathrm{mg} / \mathrm{dL}$ ), MMP-3 (normal range, $<115 \mathrm{ng} / \mathrm{ml}$ ), and VEGF (normal range, 17.3-59.7 pg/ml) levels were significantly elevated in patients with PMR before the treatment (Table 1). Rheumatic factor (RF), antinuclear antibody (ANA), myeloperoxidase-antineutrophil cytoplasmic antibody (MPO-ANCA), and proteinase-3 (PR3)-ANCA were negative in all PMR patients. Daily administration of $20 \mathrm{mg}$ corticosteroid ameliorated symptoms and significantly decreased serum CRP and VEGF levels (Table 1). Although a statistically significant difference between values before and after the treatment was not observed, corticosteroid increased serum MMP-3 levels. Nonsteroidal anti-inflammatory drugs (NSAIDs) did not ameliorate patients' symptoms and serum CRP levels (Table 2).

\section{Case A}

An 83-year-old man was admitted via emergency room complaining of fever and polyarthralgia, including bilateral wrists, knees, and hip joints. His body temperature was $38.1{ }^{\circ} \mathrm{C}$. Physical examination showed swelling of wrists, knees, and
$\mathbf{P}$

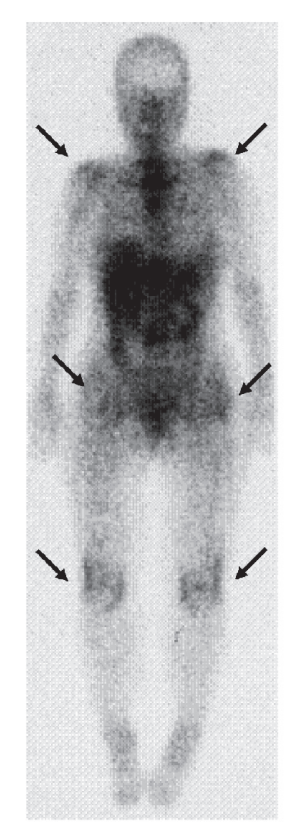

B

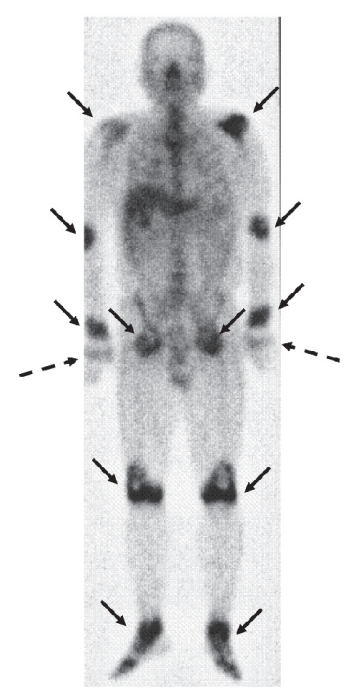

A

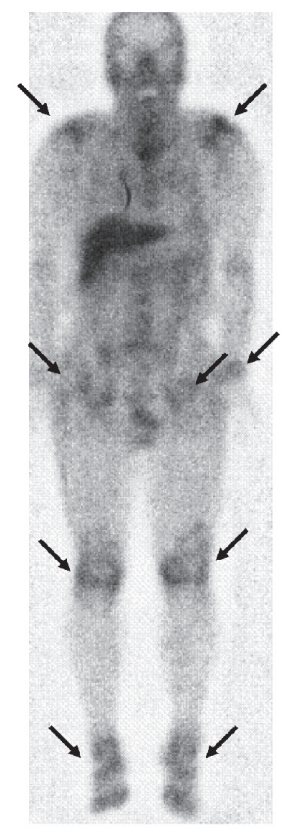

C

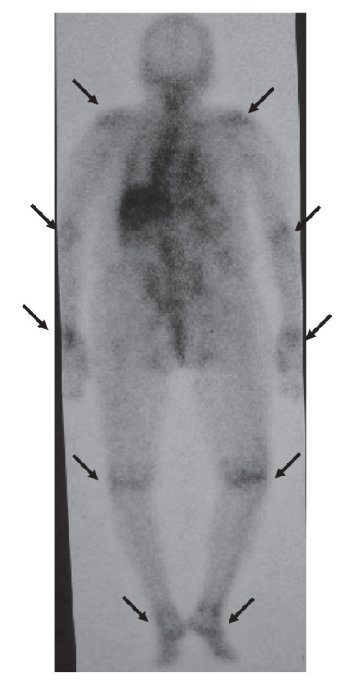

Figure I Gallium-67 scintigraphic findings of patients with polymyalgia rheumatica (P), pseudogout $\mathbf{A})$, remitting seronegative symmetrical synovitis with pitting edema (RS3PE) syndrome $\mathbf{B}$ ), and post-infectious polyarthritis $\mathbf{C}$ ). A-C corresponds to case A-C in Table I, respectively.Arrows indicate a gallium uptake. Dotted arrows indicate a gallium uptake in metacarpophalangeal joints in a patient in RS3PE syndrome.

hip joints. Laboratory evaluation on admission was notable for a CRP level of $24.3 \mathrm{mg} / \mathrm{dl}$, and RF, ANA, MPO-ANCA, and PR3-ANCA were negative. Serum MMP-3 and VEGF levels were elevated before the treatment (Table 1). His clinical and biochemical characteristics, and symmetrical and bilateral gallium uptake in shoulders, pelvic girdle, knees, and ankles, suggested the presence of PMR (Figure 1A). However, the radiographs of the wrist (Figure 2A), knee (Figure 2B), and hip (Figure 2C) showed chondrocalcinosis. 
Table I Age, serum CRP, MMP-3,VEGF levels before and after the treatment in patients with PMR, pseudogout, RS3PE syndrome, and post-infectious polyarthritis

\begin{tabular}{|c|c|c|c|c|c|c|}
\hline Case & Diagnosis & Age & & CRP (mg/dl) & MMP-3 (ng/ml) & VEGF (pg/ml) \\
\hline & PMR $(n=7)$ & $78.1 \pm 8.2$ & Before & $1 \mathrm{I} .4 \pm 4.8$ & $189.0 \pm 91.4$ & $I, I \mid 4.2 \pm 297.4$ \\
\hline & & & After & $1.5 \pm 1.0 *$ & $286.9 \pm 204.0$ & $565.1 \pm 182.3^{*}$ \\
\hline \multirow[t]{2}{*}{ A } & Pseudogout & 83 & Before & 24.3 & 252.0 & $\mathrm{I}, 190$ \\
\hline & & & After & 1.2 & 117.0 & 894 \\
\hline \multirow[t]{2}{*}{ B } & RS3PE syndrome & 65 & Before & 17.8 & 729.0 & 1,650 \\
\hline & & & After & 8.5 & 328.0 & 1070 \\
\hline \multirow[t]{2}{*}{ C } & Post-infectious polyarthritis & 89 & Before & 12.1 & 238.0 & 805 \\
\hline & & & After & 1.3 & 70.1 & 450 \\
\hline
\end{tabular}

Notes: Cases A-C corresponds to A-C in Figure I, respectively. Values in PMR patients indicate mean \pm SD. $* P<0.05$ vs values before the treatment by Wilcoxon's signed rank test. Normal range of CRP, MMP-3, and VEGF levels is $<0.3 \mathrm{mg} / \mathrm{dl},<115 \mathrm{ng} / \mathrm{ml}$, and $17.3-59.7 \mathrm{pg} / \mathrm{ml}$, respectively.

Abbreviations: CRP, C-reactive protein; MMP-3, matrix metalloproteinase-3; PMR, polymyalgia rheumatica; RS3PE, remitting seronegative symmetrical synovitis with pitting edema.

Arthrocentesis on the right knee was performed. Calcium pyrophosphate dihydrate crystals were detected in the joint fluid, suggesting that this patient was having pseudogout. Loxoprofen (180 mg/day), NSAIDs, promptly ameliorated his symptoms and decreased serum CRP level $(1.2 \mathrm{mg} / \mathrm{dl}$ at seven days after the treatment started) (Table 2), and also decreased serum MMP-3 and VEGF levels.

\section{Case B}

A 65-year-old man was diagnosed as having deep venous thrombosis (DVT) because of edema and pain in lower extremities by a family physician, and was referred to the department of cardiology. The cardiologist found no evidence of DVT and elevated serum CRP level, and then this patient was referred and admitted to our department. He complained of fever, polyarthralgia, and pitting edema in bilateral hands and legs. A gallium-67 scintigraphy revealed symmetrical uptake in shoulders, elbows, wrists, pelvic girdle, knees, and ankles (Figure 1B). In contrast to PMR, a symmetrical gallium uptake in MCP joints was observed. Serum MMP-3 and VEGF levels were significantly elevated (Table 1), and RF, ANA, MPO-ANCA, and PR3-ANCA were negative. He was finally diagnosed as having RS3PE syndrome. NSAIDs did not ameliorate patient's symptoms and serum CRP, however, a low dose of steroid (daily use of $20 \mathrm{mg}$ prednisolone) was very effective in relieving his symptoms and decreasing serum CRP level. Pitting edema on the back of hands and symmetric gallium uptake in MCP joints are characteristic for RS3PE syndrome.

\section{Case C}

An 89-year-old woman was admitted to the department of ophthalmology due to left intraocular inflammation, and her left eyeball was extracted. At the fourth day after eyeball extraction, she had fever $\left(39.2^{\circ} \mathrm{C}\right)$, and her serum CRP level was elevated $(14.5 \mathrm{mg} / \mathrm{dl})$. Antibiotic therapy was started, but, antibiotic did not decrease her body temperature and serum CRP level $(12.1 \mathrm{mg} / \mathrm{dl}$ at the fifth day after the beginning of antibiotic therapy), and she was referred and admitted to our department. She complained of fever and polyarthralgia. Serum RF, ANA,

Table 2 Changes in serum CRP levels after one week's treatment using NSAIDs in patients with PMR, pseudogout, RS3PE syndrome, and post-infectious polyarthritis

\begin{tabular}{lllll}
\hline Case & Diagnosis & & CRP $(\mathbf{m g} / \mathbf{d} \mathbf{)}$ & \%Changes of serum CRP levels \\
\hline & PMR $(\mathrm{n}=7)$ & Before & $10.4 \pm 4.4$ & $-7.2 \%$ \\
A & & After one week & $9.1 \pm 3.4^{\#}$ & $-95.1 \%$ \\
& Pseudogout & Before & 24.3 & $-22.1 \%$ \\
B & RS3PE syndrome & After one week & 1.2 & $-89.3 \%$ \\
& & Before & 20.8 & 16.2 \\
\hline
\end{tabular}

Notes: Case A-C corresponds to A-C in Figure I, respectively.Values in PMR patients indicate mean \pm SD. \#Indicates no significant differences vs values before treatment by Wilcoxon's signed rank test.

Abbreviations: CRP, C-reactive protein; MMP-3, matrix metalloproteinase-3; PMR, polymya rheumatica; RS3PE, remitting seronegative symmetrical synovitis with pitting edema. 
A

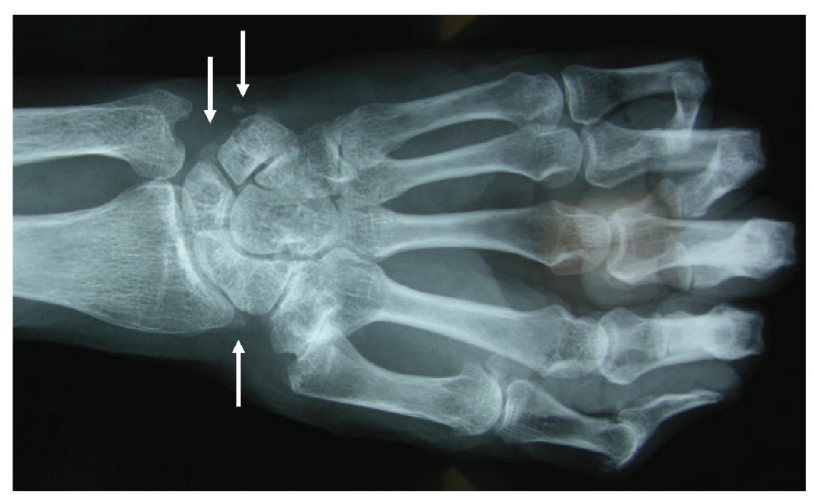

B

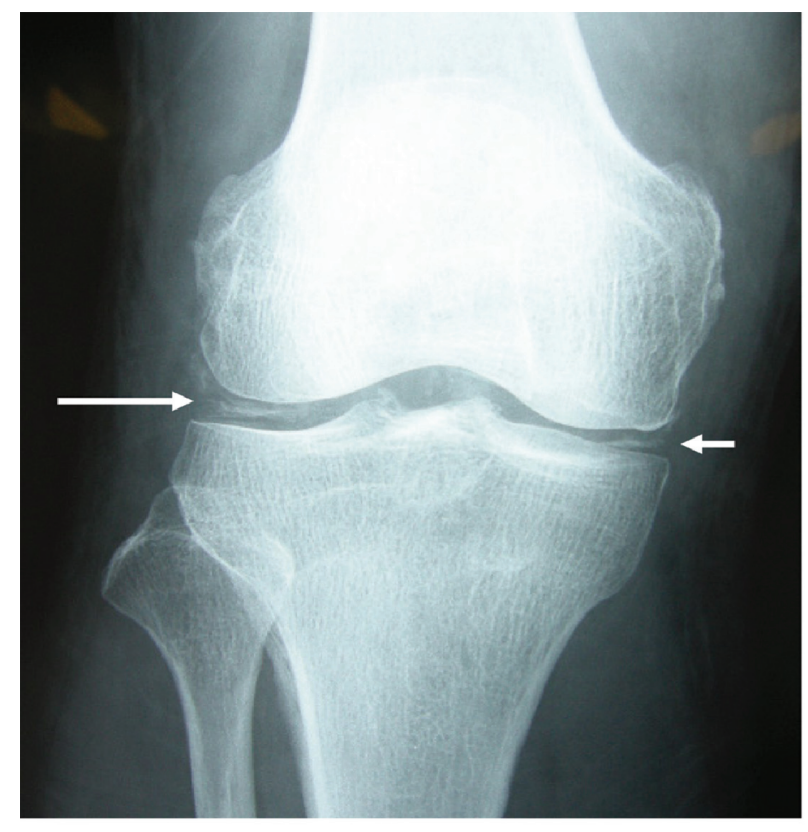

C

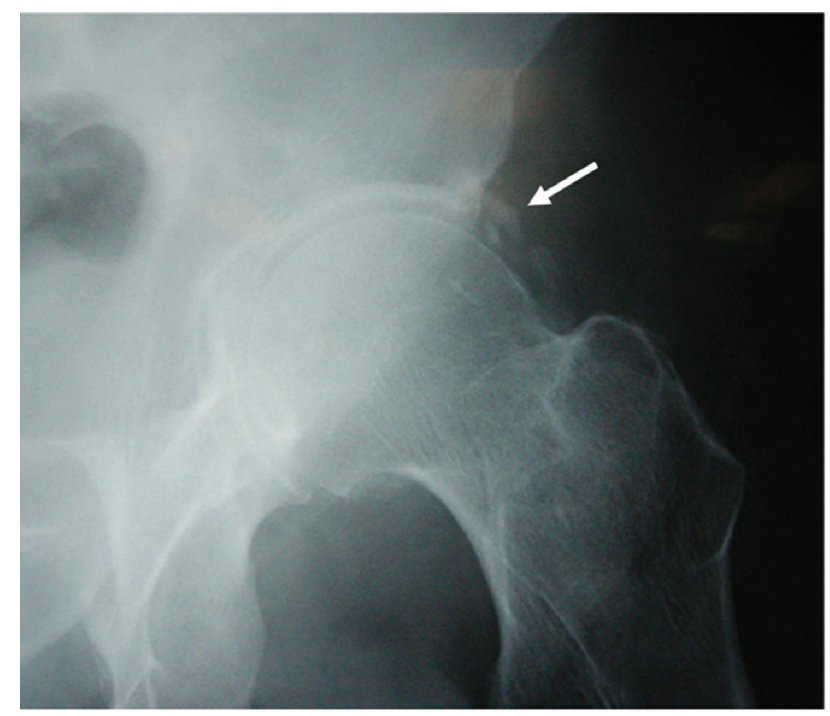

Figure 2 The radiographs of the patient with pseudogout demonstrated chondrocalcinosis (arrows) on the wrist $\mathbf{A}$ ), knee B), and hip $\mathbf{C}$ ).
MPO-ANCA, and PR3-ANCA were negative. Symmetrical gallium uptake in shoulders, elbows, wrists, knees, and ankles in a gallium-67 scintigraphy (Figure 1C) and elevated serum MMP-3 and VEGF levels (Table 1) suggested the presence of PMR. We stopped use of antibiotics and started to use loxoprofen (60-120 mg/day), NSAIDs. Loxoprofen promptly ameliorated her symptoms and decreased serum CRP level $(1.3 \mathrm{mg} / \mathrm{dl}$ at the seventh day after the treatment started). Her clinical course suggested that she was having post-infectious polyarthritis due to intraocular inflammation.

\section{Discussion}

PMR is a common disease in the elderly. In the United Kingdom, age-adjusted incidence of diagnosed PMR was reported to have increased by $35 \%$ between 1990 and $2001 .{ }^{6}$ However, it is not clear whether this suggests a true increase, increased recognition, or overdiagnosis of PMR. There is considerable uncertainty related to diagnosis in patients with PMR. ${ }^{7}$

Recently, developing classification criteria for PMR has been reported. ${ }^{8}$ Most survey respondents agreed on the importance of seven core criteria. These were 1) age $\geq 50$ years, 2) duration $\geq 2$ weeks, 3) bilateral shoulder and/or pelvic girdle aching, 4) duration of morning stiffness $>45 \mathrm{~min}, 5$ ) elevated erythrocyte sedimentation rate, 6) elevated CRP, and 7) rapid steroid response. All other core criteria except rapid steroid response were observed in patients with pseudogout and post-infectious polyarthritis, and all core criteria were observed in a patient with RS3PE syndrome, suggesting that this newly developed classification cannot distinguish pseudogout, post-infectious polyarthritis, and RS3PE syndrome from PMR.

Clinical and biochemical characteristics for PMR that we newly found were shown in Table 3. Patients with pseudogout, RS3PE syndrome, post-infectious polyarthritis manifested similar changes in scintigraphic findings and serum CRP, MMP-3, and VEGF to PMR. A good response to NSAIDs is an effective clue to differentiate pseudogout and post-infectious polyarthritis from PMR. ${ }^{9,10}$ An abnormality (chondrocalcinosis) in the radiographs of joints is also effective to differentiate pseudogout from PMR. Serum CRP level $(24.3 \mathrm{mg} / \mathrm{dl})$ in our patient (Case A) with pseudogout was quite high. A Korean female patient of calcium pyrophosphate dihydrate crystal deposition disease (CPDD) presenting as an acute polyarthritis also showed significantly elevated serum CRP levels $(18.8 \mathrm{mg} / \mathrm{dl}) .{ }^{11}$ However, it is too difficult to differentiate CPDD and CPDD + PMR from PMR, especially in elderly patients. ${ }^{12}$ Therefore, we cannot completely deny a possible existence of an additional inflammatory condition including PMR in Case A. 
Table 3 Clinical and biochemical characteristics for polymyalgia rheumatic

Age $\geq 50$ years

Elevated serum CRP, MMP-3, and VEGF levels

Symmetrical gallium-67 uptake in shoulders and/or pelvic girdle

A bad response to NSAIDs

A rapid response to steroid

No abnormalities in the radiographs of joints

Abbreviations: CRP,C-reactive protein;MMP-3, matrix metalloproteinase-3;NSAIDs, nonsteroidal anti-inflammatory drugs.

A significant difference in gallium scintigraphic findings in RS3PE syndrome as compared with PMR was the presence of gallium uptake in both MCP joints. However, symmetrical gallium uptake was observed in shoulders and pelvic girdle, showing the similar scintigraphic findings to PMR. RS3PE syndrome is a very rare inflammatory arthritis. ${ }^{13}$ The features of this syndrome are bilateral pitting edema of both hands, sudden onset of polyarthritis, age $>50$ years old, seronegative for RF. ${ }^{14}$ Mild to moderate synovitis of the joints and an association of VEGF with the pathogenesis have been observed in RS3PE syndrome as well as PMR. ${ }^{5,15,16}$ Elevated serum CRP, MMP-3 and VEGF levels, a bad response to NSAIDs, and rapid steroid response, were observed in our patient with RS3PE syndrome as well as patients with PMR. Pitting edema of the back of hands and symmetric gallium uptake in MCP joints are characteristic for RS3PE syndrome. As neoplasm are common in RS3PE syndrome, but not in PMR. Follow-up evaluation has been suggested to be necessary to observe for the possible occurrence of associated neoplasms. ${ }^{17}$

In conclusion, pseudogout, RS3PE syndrome, post-infectious polyarthritis should be included in the spectrum of diseases mimicking PMR. A good response to NSAIDs is a good clue to differentiate pseudogout and postinfectious polyarthritis from PMR. Pitting edema of the back of hands and symmetric gallium uptake in MCP joints are characteristic for RS3PE syndrome.

\section{Disclosures}

The authors report no conflicts of interest in this work.

\section{References}

1. Salvarani C, Hunder GG. Musculoskeletal manifestations in a population-based cohort of patients with giant cell arteritis. Arthritis Rheum. 1999;42:1259-1266.

2. Yanai H, Furutani N, Ito K, Yoshida H, Tada N. Scintigraphic findings and serum matrix metalloproteinase 3 and vascular endothelial growth factor levels in patients with polymyalgia rheumatica. The Open General and Internal Medicine Journal. 2009;3:53-57.

3. Chuang TY, Hunder GG, Ilstrup DM, Kurland LT. Polymyalgia rheumatica: a 10-year epidemiologic and clinical study. Ann Intern Med. 1982;97:672-680.

4. Ribbens C, Martin y Porras M, Franchimont N, et al. Increased matrix metalloproteinase-3 serum levels in rheumatic diseases: relationship with synovitis and steroid treatment. Ann Rheum Dis. 2002;61: $161-166$.

5. Meliconi R, Pulsatelli L, Dolzani P, et al. Vascular endothelial growth factor production in polymyalgia rheumatica. Arthritis Rheum. 2000;43:2472-2480.

6. Smeeth L, Cook C, Hall AJ. Incidence of diagnosed polymyalgia rheumatica and temporal arteritis in the United Kingdom, 1990-2001. Ann Rheum Dis. 2006;65:1093-1098.

7. Dasgupta B, Hutchings A, Matteson EL. Polymyalgia rheumatica: the mess we are now in and what we need to do about it. Arthritis Rheum. 2006;55:518-520

8. Dasgupta B, Salvarani C, Schirmer M, et al. Developing classification criteria for polymyalgia rheumatica: comparison of views from an expert panel and wider survey. J Rheumatol. 2008;35:270-277.

9. Petersel DL, Sigal LH. Reactive arthritis. Infect Dis Clin North Am. 2005;19:863-883.

10. Announ N, Guerne PA. Treating difficult crystal pyrophosphate dihydrate deposition disease. Curr Rheumatol Rep. 2008;10:228-234.

11. Song JS, Lee YH, Kim SS, Park W. A case of calcium pyrophosphate dihydrate crystal deposition disease presenting as an acute polyarthritis. J Korean Med Sci. 2002; 17:423-425.

12. Pego-Reigosa JM, Rodriguez-Rodriguez M, Hurtado-Hernandez Z, et al. Calcium pyrophosphate deposition disease mimicking polymyalgia rheumatica: a prospective followup study of predictive factors for this condition in patients presenting with polymyalgia symptoms. Arthritis Rheum. 2005;53:931-938.

13. McCarty DJ, O’Duffy JD, Pearson L, Hunter JB. Remitting seronegative symmetrical synovitis with pitting edema. RS3PE syndrome. JAMA. $1985 ; 254: 2763-2767$

14. Olive A, del Blanco J, Pons M, Vaquero M, Tena X. The clinical spectrum of remitting seronegative symmetrical synovitis with pitting edema. The Catalan Group for the Study of RS3PE. J Rheumatol. 1997;24:333-336.

15. Pulsatelli L, Dolzani P, Silvestri T, et al. Synovial expression of vasoactive intestinal peptide in polymyalgia rheumatica. Clin Exp Rheumatol. 2006;24:562-566.

16. Arima K, Origuchi T, Tamai M, et al. RS3PE syndrome presenting as vascular endothelial growth factor associated disorder. Ann Rheum Dis. 2005;64:1653-1655.

17. Triplett LM, Pfister AK. The clinical entity of remitting seronegative synovitis with pitting edema (RS3PE syndrome). $W \mathrm{~V} \mathrm{Med} \mathrm{J}$ 2001;97:302-304
Clinical Interventions in Aging

\section{Publish your work in this journal}

Clinical Interventions in Aging is an international, peer-reviewed journal focusing on evidence-based reports on the value or lack thereof of treatments intended to prevent or delay the onset of maladaptive correlates of aging in human beings. This journal is indexed on PubMed Central, MedLine, the American Chemical Society's 'Chemical

\section{Dovepress}

Abstracts Service' (CAS), Scopus and the Elsevier Bibliographic databases. The manuscript management system is completely online and includes a very quick and fair peer-review system, which is all easy to use. Visit http://www.dovepress.com/testimonials.php to read real quotes from published authors. 